\title{
The influence of collisional-ionization and recombination processes on spectral line shapes in stellar atmospheres and in the hydrogen clouds in broad-line region of AGNs
}

\author{
V.A. Srećković ${ }^{1}$, M.S. Dimitrijević ${ }^{2,3}$ and Lj.M. Ignjatović ${ }^{1}$ \\ 1 Institute of Physics Belgrade, Pregrevica 118, 11080 Zemun, Belgrade, \\ Serbia, (E-mail:vlada@ipb.ac.rs) \\ 2 Astronomical Observatory, Volgina 7, 11060 Belgrade, Serbia, (E-mail: \\ mdimitrijevic@aob.rs) \\ 3 Sorbonne Université, Observatoire de Paris, Université PSL, CNRS, \\ LERMA, F-92190, Meudon, France
}

Received: July 16, 2019; Accepted: August 22, 2019

\begin{abstract}
In this paper, the importance of $\mathrm{H}^{*}(n)+\mathrm{H}(1 s)$ collisional ionization as well as the influence of inverse recombination processes in different environments has been investigated within the regions $2 \leq n \leq 20$ and 4000 $\mathrm{K} \leq T \leq 20000 \mathrm{~K}$. The interpretation of this influence is based on existing method of describing inelastic processes in symmetrical atomRydberg-atom collisions. These processes have effect on ionization and the populations of hydrogen excited atoms in moderately ionized plasma layers. From the results it follows that the investigation of these processes is of interest for the research and modelling of such medium.
\end{abstract}

Key words: Atomic processes - Galaxies: active - Line: profiles - Plasmas

\section{Introduction}

The processing of line spectra with radiative transfer calculations requires spectroscopic data as well as collisional data (e.g., cross sections, rate coefficients, atomic parameters, etc Baron \& Hauschildt 1998; Mihajlov et al. 2011a; Ignjatović et al. 2014; Srećković et al. 2018a). The fact that the ionization processes which involve highly excited atoms (Mihajlov et al., 2012; Srećković et al., 2017) influence the ionization level and atom excited-state populations, could influence the optical properties of the weakly ionized regions (Mihajlov et al., 2011b) of very dense parts of the clouds in broad line region (BLR) of active galactic nuclei (AGN), and potentially be important for the spectroscopy and modeling of such environment (Crosas \& Weisheit, 1993; Osterbrock \& Ferland, 2006).

Consequently, it is essential to find out at what plasma conditions such processes become important and to eliminate uncertainties of the rate coefficients 
due to hydrogen collisions (Barklem, 2007; Srećković et al., 2018a), so that they can be properly included in numerical simulations (Ferland et al., 1998, 2017).

If an atom is in a state of sufficiently high principal quantum number $n$ it means that the valence electron is far from the ionic core, and such atom appears hydrogenic. It is commonly accepted to call such atoms as Rydberg atoms with electron excited into a high lying Rydberg state, with large principal quantum number. For Rydberg atoms even inelastic thermal collisions can be sufficiently energetic to lead to ionization reactions (Gnedin et al., 2009; Mihajlov et al., 2012). These types of reactions are collisional ionization processes of associative ionization and the non-associative ionization

$$
\mathrm{H}^{*}(n)+\mathrm{H} \Leftrightarrow\left\{\begin{array}{l}
\mathrm{H}_{2}^{+}+e \\
\mathrm{H}+\mathrm{H}^{+}+e
\end{array}\right.
$$

and the corresponding inverse recombination processes where $\mathrm{H}^{*}(n)$ represents a high $n$ Rydberg state of an atom $\mathrm{H}$ and e is a free electron, where $\mathrm{H}=\mathrm{H}(1 \mathrm{~s})$. Here the processes (1) and (2) are theoretically considered as factors of influence on the populations of excited atoms in the weakly ionized hydrogen plasmas. It means that the efficiency of these processes should be compared with the efficiency of the collisional processes $\mathrm{H}^{*}(n)+e \Leftrightarrow \mathrm{H}^{+}+2 e$ and $\mathrm{H}^{+}+e \Rightarrow$ $\mathrm{H}^{*}(n)+\varepsilon_{\lambda}$. For the considered conditions of weakly ionized hydrogen plasmas the rate coefficients for collisional ionisation and collisional recombination processes are calculated as well.

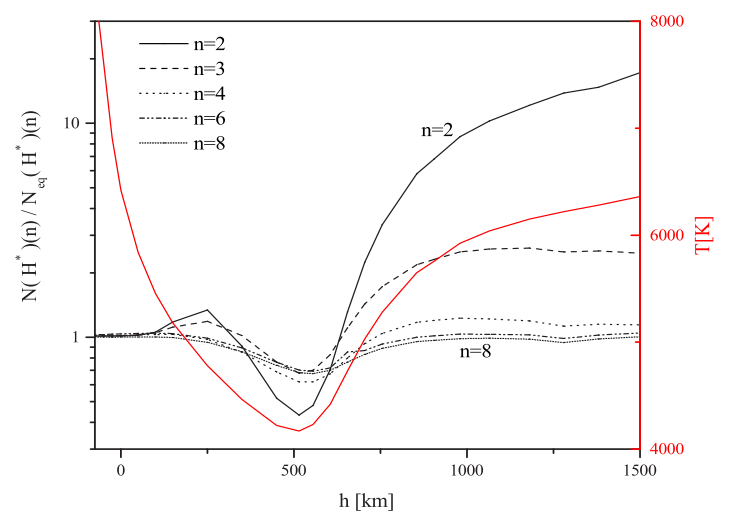

Figure 1. The ratio $N\left(\mathrm{H}^{*}\right)(n) / N\left(\mathrm{H}_{e q}^{*}\right)(n)$, as a function of height h. The index "eq" denotes that excited atom densities correspond to thermodynamical equilibrium conditions for given $T$. 
Let us note the fact concerning (1) and (2) collision processes. Namely the considered processes strongly influence to the excited state populations and the free electron density (Mihajlov et al., 2011b). As the consequence of these facts, the significant change of the shape of the hydrogen atomic spectral lines is expected in different environments (see e.g. Gnedin et al. 2009). Also, let us emphasize the fact that the importance of these processes for non-local thermodynamic equilibrium (LTE) modeling of different atmosphere should be further investigated. In Fig. 1, deviations of non-LTE populations of excited hydrogen atom states with $2 \leq n \leq 8$, in solar photosphere are illustrated (see Mihajlov et al. 2011b). One can see that these deviations are particularly pronounced for $\mathrm{n}=2$. This deviation is noticeable, illustrating the importance of taking into account the considered processes ab initio in the modeling.

\section{The collisional ionisation/recombination data}

As in the previous papers (Mihajlov et al., 2011b; Mihajlov et al., 2012), for the hydrogen Rydberg states $(n \gg 1)$ we will treat processes $(1)$ and $(2)$ on the basis of dipole resonant mechanism. Such processes are considered as a result of resonant energy conversion within the electronic component of the collisional system $\mathrm{H}^{*}(n)+\mathrm{H}(1 s)$, which is realized inside the region $R<<r_{n}$, where the collisional system can be presented as $\left[\mathrm{H}^{+}+\mathrm{H}(1 s)\right]+e_{n}$, and which is caused by the dipole part of the interaction of the outer electron $e_{n}$ with the subsystem $\left[\mathrm{H}^{+}+\mathrm{H}(1 s)\right]$. The parameter $r_{n} \sim n^{2}$ is the characteristic radius of Rydberg atom $\mathrm{H}^{*}(n)$ and $R$ is the inter-nuclear distance in the collision system $\mathrm{H}^{*}(n)+\mathrm{H}(1 s)$. The processes (1) and (2) for the $2 \leq n \leq 4$ will be considered separately as in Mihajlov et al. (2011b), due to the behaviour of the adiabatic potential curves of atom-atom collisional systems. Here we presented a brief description of the method with the basic theory (for details, see Mihajlov et al. 2012; O'Keeffe et al. 2012).

The aim of this work is to find out at what plasma conditions collisional ionisation/recombination become important and to update values and eliminate uncertainties of the rate coefficients due to hydrogen collisions (Barklem, 2007; Srećković et al., 2018a) in order that they can be properly included in numerical simulations and modelling (Ferland et al., 1998, 2017) as well as in the A\&M databases (Vujčić et al., 2015; Dubernet et al., 2016; Marinković et al., 2017).

The corresponding partial rate coefficients of the collisional ionisation/ recombination processes $(1)$ and $(2)$ are denoted here with $K_{c i, c r}^{(a, b)}(n ; T)$, where $T$ is the temperature of the considered plasma. Using these partial rate coefficients we determined the total ones $K_{c i, c r}(n, T)$, namely,

$$
K_{c i, c r}(n, T)=K_{c i, c r}^{(a)}(n, T)+K_{c i, c r}^{(b)}(n, T),
$$

which characterizes the efficiency of the considered collisional processes together (for details see Mihajlov et al. 2011b). Here we will consider collisional ionisa- 
tion/ recombination processes within the regions $2 \leq n \leq 20$ and $4000 \mathrm{~K} \leq T \leq$ $20000 \mathrm{~K}$. Relative contribution of associative and non-associative channels i.e.
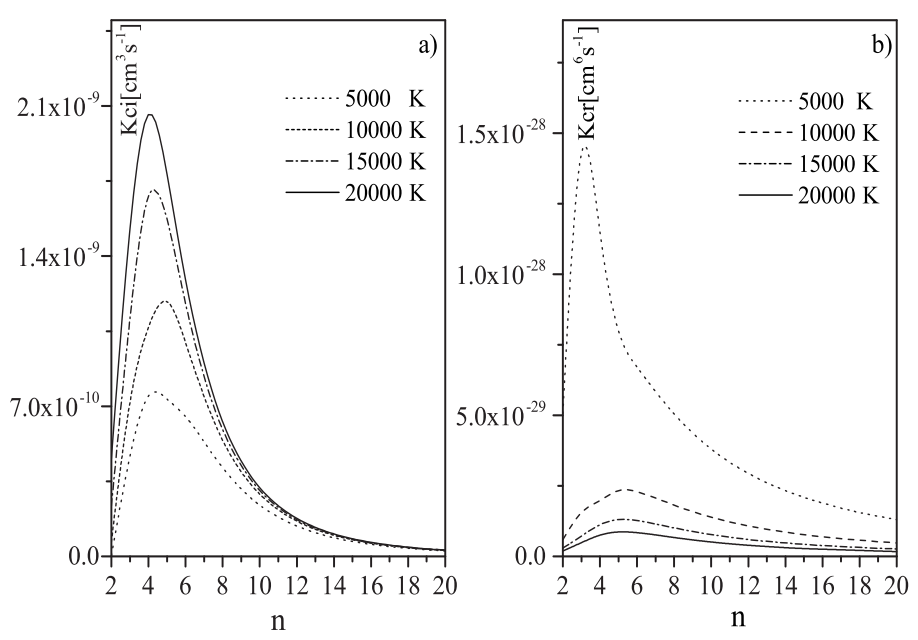

Figure 2. The calculated values of rate coefficients for collisional ionization and recombination $K_{c i}(n, T)$ and $K_{c r}(n, T)$.

partial collisional ionisation/ recombination for given $n$ and $T$ can bee characterized by corresponding branch factor i.e. ratio $K_{c i}^{(a, b)}(n, T) / K_{c i}(n, T)$. This provides important information about presence of molecular ion $\mathrm{H}_{2}^{+}$.

The total collisional ionization $I_{c i}(n, T)$, and collisional recombination $I_{c r}(n, T)$ fluxes caused by the processes (1) and (2), can be expressed as,

$$
I_{c i}(n, T)=K_{c i}(n, T) \cdot N_{n} N_{1}, \quad I_{c r}(n, T)=K_{c r}(n, T) \cdot N_{1} N_{i} N_{e} .
$$

This is performed under the standard assumption that in e.g. photosphere plasmas $T_{e}=T_{a}=T$, where $T_{e}$ and $T_{a}$ are the electron and atom temperatures and $\mathrm{T}$ is their common value. Let $I_{i ; e a}(n, T)=K_{e a}(n, T) \cdot N_{n} N_{e}, I_{r ; e e i}(n, T)=$ $K_{e e i}(n, T) \cdot N_{i} N_{e} N_{e}$ and $I_{r ; p h}(n, T)=K_{p h}(n, T) \cdot N_{i} N_{e}$ are the fluxes caused by ionization and recombination processes where $N_{1}, N_{n}, N_{i}$, and $N_{e}$ are, respectively, the densities of the ground and excited states of a hydrogen atom, of ion, and of free electron in the considered plasma with given $T$.

Using these expressions, we can calculate quantities i.e. flux ratio $\frac{I_{c i}(n, T)}{I_{i: e a}(n, T)}$ which characterize the relative efficiency of partial ionization processes (1) and (2) together and the impact electron-atom ionization in the considered plasma. 


\section{Results and Discussion}

The values of the total ionization and recombination rate coefficients $K_{c i}(n, T)$ and $K_{c r}(n, T)$, obtained in the described way, are presented in Fig. 2. These results cover the regions $2 \leq n \leq 20$ and $4000 \mathrm{~K}<T \leq 20000 \mathrm{~K}$ which are relevant for moderately ionized plasma layers in different environments.

In order to allow for a faster usage of the calculated results we provide a fit to numerical results of the form (Sahal-Bréchot et al., 2014)

$$
\log \left(K_{c i, c r}(T)\right)=\sum_{i=0}^{2} a_{i}(\log (T))^{i}
$$

The fits are valid over the temperature range of $4000 \mathrm{~K} \leq T \leq 20000 \mathrm{~K}$ (see Mihajlov et al. (2011a); Srećković et al. (2018a); Srećković et al. (2018b)). This enables the easy inclusion of these processes in the modeling not only the very dense parts of the clouds in AGN BLR but also moderately ionized layers of the Sun and solar like stars. The parameters are provided in Tab. 1 for selected excited states $(\mathrm{n}=4,5)$.

Table 1. The fits of the Eq. (5) to the rate coefficients (Mihajlov et al., 2011a; Srećković et al., 2018a). A portion is shown here for guidance regarding its form and content.

\begin{tabular}{cccc}
\hline \hline $\mathrm{n}$ & $a_{0}$ & \multicolumn{2}{c}{$a_{1}$} \\
\hline & \multicolumn{3}{c}{$K_{c i}$} \\
\cline { 2 - 4 } 4 & 19.91758 & -15.14785 & 1.98009 \\
5 & -20.60455 & 5.21174 & -0.57122 \\
& & $K_{c r}$ & \\
\cline { 2 - 4 } 4 & 35.17003 & -30.09052 & 3.52731 \\
5 & -15.84169 & -4.85018 & 0.41418 \\
\hline
\end{tabular}

These results, and further analysis show that associative channel is dominant for lower $n$ and $T$. This provides important information about presence of molecular ion $\mathrm{H}_{2}^{+}$. The importance of associative channel decreases with temperature increase when non-associative channel take dominant place.

In Fig. 3 the behavior of flux ratio $\frac{I_{c i}(n, T)}{I_{i ; e a}(n, T)}$ for $2 \leq n \leq 8$, for the plasma condition with neutral hydrogen atom in the ground state densities $10^{13} \mathrm{~cm}^{-3}$ and the electron density of $0.01 N_{1}$, at $T=8000 \mathrm{~K}$, is shown. For $2 \leq n \leq 5$ the efficiency of the considered collisional ionization processes is at least comparable with the electron-atom impact ionization. The same conclusion goes for influence of the collisional recombination processes together on the same block of excited hydrogen atom states. 


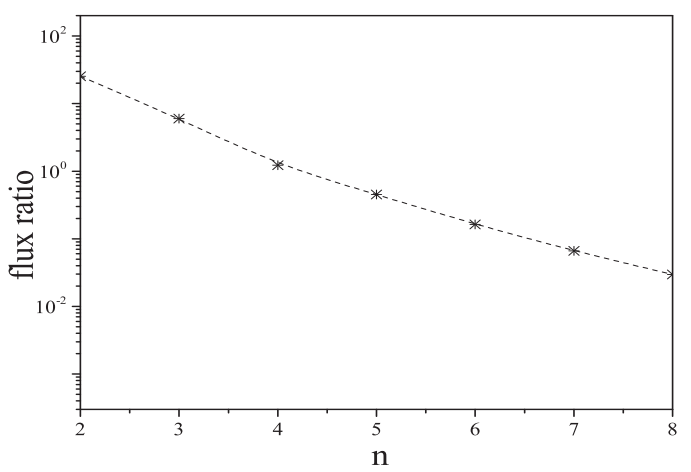

Figure 3. Behavior of the quantity i.e. flux ratio $\frac{I_{c i}(n, T)}{I_{i ; e a}(n, T)}$ as a function of $n$, for the neutral hydrogen atom ground state densities $N_{1}=10^{13} \mathrm{~cm}^{-3}$ and temperatures $T=8000 \mathrm{~K}$ with the electron density $0.01 N_{1}$.

Analyzing the flux ratio the influence of investigated processes increases linearly with hydrogen density, and, for example for $10^{13} \mathrm{~cm}^{-3}$ some optical characteristics may be different than for $10^{12} \mathrm{~cm}^{-3}$, due to changes in electron density, energy level populations, formation of hydrogen molecules, etc. In future it will be very useful to do an analysis (e.g with the code CLOUDY) in order to see changes in optical characteristics.

About this issue, in Mihajlov et al. (2007) was examined the atmosphere of a $\mathrm{M}$ red dwarf with an effective temperature of $3800 \mathrm{~K}$. For the examined atmosphere, the profiles of a number of spectral lines of the hydrogen atom were calculated (see also Gnedin et al. (2009)). Profiles are synthesized with PHOENIX code with Stark broadening contribution. Analyses show that the collisional-ionization/recombination processes, influence on the population of the hydrogen excited states and the electron densities, and have a very strong effect on the shape of the spectral lines (see also Gnedin et al. 2009; Srećković et al. 2018a).

\section{Conclusions}

The presented data and analyses demonstrate the fact that the considered ionization/recombination processes, influence on the ionization level and atom excited-state populations and have a noticeable influence on the optical properties of very dense parts of the clouds in the BLR of AGNs where the neutral hydrogen densities are larger than $10^{12} \mathrm{~cm}^{-3}$ since in such conditions they dominate over the relevant concurrent electronatom collision processes. The calculated values of the rate coefficients are also very useful for the modelling and 
analysis of similar layers in the photospheres of Sun and solar like stars and laboratory plasmas (Barklem, 2007; Fontenla et al., 2009) and for the study of their influence during the cosmological recombination epoch (Chluba et al., 2010). Note that the obtained results point to further directions of the investigation. In future it will be very useful to do an analysis with the code CLOUDY in order to see changes in optical characteristics i.e. on the line shapes.

Acknowledgements. This work is made within projects of the MESTD of the Republic of Serbia Grants OI176002, III44002.

\section{References}

Barklem, P. S., Non-LTE Balmer line formation in late-type spectra: effects of atomic processes involving hydrogen atoms. 2007, Astron. Astrophys., 466, 327

Baron, E. \& Hauschildt, P. H., Parallel Implementation of the PHOENIX Generalized Stellar Atmosphere Program. 1998, Astrophys. J., 495, 370

Chluba, J., Vasil, G. M., \& Dursi, L. J., Recombinations to the Rydberg states of hydrogen and their effect during the cosmological recombination epoch. 2010, Mon. Not. R. Astron. Soc., 407, 599

Crosas, M. \& Weisheit, J. C., Hydrogen molecules in quasar broad-line regions. 1993, Mon. Not. R. Astron. Soc., 262, 359

Dubernet, M. L., Antony, B. K., Ba, Y. A., et al., The virtual atomic and molecular data centre (VAMDC) consortium. 2016, J. Phys. B, 49, 074003

Ferland, G. J., Chatzikos, M., Guzmán, F., et al., The 2017 Release Cloudy. 2017, Rev. Mex. Astron. Astrofis, 53, 385

Ferland, G. J., Korista, K. T., Verner, D. A., et al., CLOUDY 90: Numerical Simulation of Plasmas and Their Spectra. 1998, Publ. Astron. Soc. Pac., 110, 761

Fontenla, J. M., Curdt, W., Haberreiter, M., Harder, J., \& Tian, H. Semiempirical Models of the Solar Atmosphere. III. Set of Non-LTE Models for FarUltraviolet/Extreme-Ultraviolet Irradiance Computation. 2009, Astrophys. J., 707, 482

Gnedin, Y. N., Mihajlov, A., Ignjatović, L. M., et al., Rydberg atoms in astrophysics. 2009, New Astron. Rev., 53, 259

Ignjatović, L. M., Mihajlov, A., Srećković, V., \& Dimitrijević, M., Absorption nonsymmetric ion-atom processes in helium-rich white dwarf atmospheres. 2014, Mon. Notices Royal Astron. Soc., 439, 2342

Marinković, B. P., Jevremović, D., Srećković, V. A., et al., BEAMDB and MolDdatabases for atomic and molecular collisional and radiative processes: Belgrade nodes of VAMDC. 2017, Eur. Phys. J. D, 71, 158

Mihajlov, A. A., Jevremović, D., Hauschildt, P., et al., Influence of chemi-ionization and chemi-recombination processes on hydrogen line shapes in M dwarfs. 2007, Astron. Astrophys., 471, 671 
Mihajlov, A., Sakan, N., Srećković, V., \& Vitel, Y., Modeling of continuous absorption of electromagnetic radiation in dense partially ionized plasmas. 2011a, J. Phys. A, 44, 095502

Mihajlov, A., Srećković, V., Ignjatović, L. M., \& Klyucharev, A., The chemi-ionization processes in slow collisions of Rydberg atoms with ground state atoms: mechanism and applications. 2012, J. Clust. Sci., 23, 47

Mihajlov, A. A., Ignjatović, L. M., Srećković, V. A., \& Dimitrijević, M. S., Chemiionization in Solar Photosphere: Influence on the Hydrogen Atom Excited States Population. 2011b, Astrophys. J., Suppl., 193, 2

O'Keeffe, P., Bolognesi, P., Avaldi, L., et al., Experimental and theoretical study of the chemi-ionization in thermal collisions of Ne Rydberg atoms. 2012, Phys. Rev. A, 85, 052705

Osterbrock, D. E. \& Ferland, G. J. 2006, Astrophysics of gaseous nebulae and active galactic nuclei (University science books)

Sahal-Bréchot, S., Dimitrijević, M. S., Moreau, N., \& Ben Nessib, N., The STARK-B database as a resource for STARK widths and shifts data: State of advancement and program of development. 2014, Adv. Space Res., 54, 1148

Srećković, V., Dimitrijević, M., Ignjatović, L., Bezuglov, N., \& Klyucharev, A., The Collisional Atomic Processes of Rydberg Hydrogen and Helium Atoms: Astrophysical Relevance. 2018a, Galaxies, 6, 72

Srećković, V. A., Dimitrijević, M. S., \& Ignjatović, L. M., Atom-Rydberg atom chemiionization/recombination processes in the hydrogen clouds in broad-line region of AGNs. 2018b, Mon. Not. R. Astron. Soc., 480, 5078

Srećković, V., Ignjatović, L., Jevremović, D., Vujčić, V., \& Dimitrijević, M., Radiative and Collisional Molecular Data and Virtual Laboratory Astrophysics. 2017, Atoms, 5, 31

Vujčić, V., Jevremović, D., Mihajlov, A., et al., MOL-D: A Collisional Database and Web Service within the Virtual Atomic and Molecular Data Center. 2015, J. Astrophys. Astron., 36, 0 